\title{
Pre-discharge anemia as a predictor of adverse clinical outcomes in patients with acute decompensated heart failure
}

Min Chul Kim ${ }^{1,2}$, Kye Hun Kim ${ }^{1}$, Jae Yeong Cho ${ }^{1}$, Ki Hong Lee ${ }^{1}$, Doo Sun Sim ${ }^{1,2}$, Hyun Ju Yoon ${ }^{1}$, Nam Sik Yoon ${ }^{1}$, Young Joon Hong ${ }^{1,2}$, Hyung Wook Park ${ }^{1}$, Ju Han Kim ${ }^{1,2}$, Youngkeun Ahn ${ }^{1,2}$, Myung Ho Jeong ${ }^{1,2}$, Jeong Gwan Cho ${ }^{1}$, and Jong Chun Park ${ }^{1}$

${ }^{1}$ Department of Cardiology, ${ }^{2}$ Specialized Research Consortium of Cardiovascular Medicine, Chonnam National University Hospital, Gwangju, Korea

Received: October 8, 2017 Revised : November 27, 2017 Accepted: December 30, 2017

\section{Correspondence to}

Kye Hun Kim, M.D.

Department of Cardiology, Chonnam National University Hospital, 42 Jebong-ro, Dong-gu, Gwangju 61469, Korea

Tel: +82-62-220-6978

Fax: +82-62-223-3105

E-mail:christiankyehun@hanmail.net
Background/Aims: The impact of the timing of anemia during hospitalization on future clinical outcomes after surviving discharge from an index heart failure (HF) has been poorly studied in patients with acute decompensated heart failure (ADHF). Methods: A total of 384 surviving patients with acute ADHF were divided into two groups: an anemia group $(\mathrm{n}=270,199$ anemia at admission and 71 pre-discharge anemia) and a no anemia group $(\mathrm{n}=114)$. All-cause mortality and HF re-hospitalization were compared between groups.

Results: During the follow-up period (median, 528 days), death occurred in 60 patients (15.6\%) and HF re-hospitalization occurred in 131 patients (34.1\%). Overall anemia was associated with increased mortality (hazard ratio [HR], 1.74; 95\% confidence interval [CI], 1.03 to 3.01; $p=0.039)$, but not $\mathrm{HF}$ re-hospitalization (HR, 0.92; $95 \%$ CI, 0.59 to $1.42 ; p=0.707$ ). Pre-discharge anemia was significantly associated with increased mortality (HR, 1.68; $95 \%$ CI, 1.01 to $2.82 ; p=0.048)$, but anemia at admission did not predict increased mortality or re-hospitalization.

Conclusions: Pre-discharge anemia, rather than anemia at admission, was identified as an independent predictor of mortality in patients with ADHF after surviving discharge. The results of the present study suggest that the identification and optimal management of anemia during hospitalization are important in patients with ADHF.

Keywords: Acute heart failure; Anemia; Prognosis

\section{INTRODUCTION}

Anemia, defined as a decreased level of blood hemoglobin $(\mathrm{Hgb})$, is common in patients with heart failure (HF). Previous studies have demonstrated that anemia is not only an important predisposing condition of HF aggravation, but also a predictor of poor prognosis in patients with acute decompensated heart failure (ADHF) [1-4].
Despite the importance of anemia in ADHF, previous studies have mostly focused on the clinical significance of anemia at admission. Few studies have investigated the associations between clinical outcomes and anemia developing at different times during hospitalization, even though anemia can onset at any time during hospitalization in association with blood loss, infection, impaired renal function, or other causes [5]. Recent studies have suggested that dynamic changes of Hgb levels are 
associated with future clinical outcomes in patients with ADHF [6-8].

We hypothesized that pre-discharge anemia may not be managed appropriately following discharge after $\mathrm{HF}$, even though anemia at admission is usually corrected during hospitalization in patients with ADHF. Therefore, there is a possibility that pre-discharge anemia, as compared to anemia at admission, may have a more significant impact on future clinical outcomes in ADHF. Consequently, we evaluated the impact of anemia that developed at any time during hospitalization on future clinical outcomes after discharge in patients with ADHF.

\section{METHODS}

\section{Study design and population}

The present study used a single-center, retrospective observational design, and the study protocol was approved by the Institutional Review Board (approval number: CNUH-2015-231) of our institution. A waiver for informed consent was obtained from the Institutional Review Board.

From May 2011 to March 2013, a total of 394 consecutive patients with ADHF were identified. After excluding 10 patients who died during hospitalization, 384 surviving patients were enrolled in the present study and divided into two groups according to the presence of anemia during hospitalization: an anemia group (presence of anemia at any time during hospitalization; $\mathrm{n}=27 \mathrm{O}$, $71.3 \pm 11.8$ years, 131 males) and a no anemia group ( $\mathrm{n}=$ $114,62.4 \pm 16.4$ years, 62 males). All patients were treated with transfusion to raise the level of $\mathrm{Hgb}$ above $7 \mathrm{~g} / \mathrm{dL}$ during hospitalization.

All-cause mortality and HF-related re-hospitalization during clinical follow-up were compared between groups. Two separate additional analyses were performed to investigate the impact of anemia at different times during hospitalization: admission anemia versus no admission anemia, and discharge anemia versus no discharge anemia.

\section{Study definitions}

ADHF was diagnosed by the combination of typical symptoms and/or signs, echocardiographic findings, and elevated levels of natriuretic peptides according to the current guidelines for HF [9-11]. The severity of ADHF was evaluated using New York Heart Association (NYHA) functional classification at the time of admission and discharge in all patients.

Serial levels of $\mathrm{Hgb}$ were checked during hospitalization, and anemia was defined according to the World Health Organization definition $(\mathrm{Hgb}<13 \mathrm{~g} / \mathrm{dL}$ in men and $<12 \mathrm{~g} / \mathrm{dL}$ in women) [12]. Admission and discharge anemia were diagnosed using the first $\mathrm{Hgb}$ value obtained during hospitalization and the last $\mathrm{Hgb}$ value before discharge, respectively. We also defined hospital-acquired anemia, which included discharge anemia, using the lowest $\mathrm{Hgb}$ value in patients without admission anemia.

\section{Echocardiography examination}

Echocardiography examinations were performed using a digital ultrasonography system (Vivid 7, GE Vingmed Ultrasound, Horten, Norway), and all data were analyzed using a computerized software package (EchoPAC PC 6.o.o, GE Vingmed Ultrasound). The protocol of our echocardiography laboratory was summarized previously [13]. Comprehensive echocardiographic studies, including Doppler studies, were performed as soon as possible after hospitalization according to the current guideline [14]. Left ventricular ejection fraction (LVEF) was measured using Simpson's method, and intra- and interobserver variation for LVEF were $4 \% \pm 5 \%$ and $5 \%$ $\pm 4 \%$, respectively (absolute difference divided by the mean of the measured value).

\section{Statistical analysis}

Continuous variables are presented as mean \pm standard deviation or as medians and interquartile ranges, and were compared using the unpaired $t$ test or the Mann-Whitney rank-sum test. Discrete variables are expressed as counts and percentages, and were analyzed with Pearson chi-square test or Fisher exact test. We constructed Kaplan-Meier curves to compare the study endpoints between the two groups, and differences were assessed with the log-rank test. Cox proportional hazards regression with adjustment for covariates was used to assess clinical outcomes. Variables with $p<0.05$ in univariate Cox regression analysis, and those known to be relevant to clinical outcomes in ADHF were included 
in the multivariate Cox regression analysis: age, systolic blood pressure $\leq 100 \mathrm{mmHg}$, NYHA classification, diabetes mellitus, ischemic etiology of ADHF, LVEF, atrial fibrillation, hyponatremia, and serum creatinine. All analyses were two-tailed, and all variables were consid- ered significant at a value of $p<0.05$. All statistical analyses were performed using SPSS for Windows software version 21.o (IBM Co., Armonk, NY, USA).

\section{Table 1. Baseline clinical characteristics}

\begin{tabular}{|c|c|c|c|}
\hline Characteristic & Anemia $(n=270)$ & No anemia $(n=114)$ & $p$ value \\
\hline Age, yr & $71.3 \pm 11.8$ & $62.4 \pm 16.4$ & $<0.001$ \\
\hline Male sex & $131(48.5)$ & $62(54.4)$ & 0.293 \\
\hline Body mass index, $\mathrm{kg} / \mathrm{m}^{2}$ & $23.2 \pm 5.1$ & $24.2 \pm 3.7$ & 0.048 \\
\hline Systolic blood pressure, mmHg & $126.8 \pm 23.4$ & $127.8 \pm 24.9$ & 0.709 \\
\hline Systolic blood pressure $\leq 100 \mathrm{mmHg}$ & $46(17.0)$ & $19(16.7)$ & 0.930 \\
\hline Diastolic blood pressure, $\mathrm{mmHg}$ & $78.6 \pm 15.1$ & $80.2 \pm 15 \cdot 3$ & 0.352 \\
\hline Heart rate, beats/min & $88.1 \pm 22.9$ & $91.2 \pm 23.8$ & 0.234 \\
\hline HF with reduced ejection fraction ${ }^{a}$ & $158(58.5)$ & $70(61.4)$ & 0.599 \\
\hline NYHA class at admission & $3.2 \pm 0.7$ & $3.0 \pm 0.8$ & 0.010 \\
\hline NYHA classification $\geq 3$ & $224(83.0)$ & $79(69 \cdot 3)$ & 0.003 \\
\hline NYHA class at discharge & $2.2 \pm 0.7$ & $2.0 \pm 0.5$ & 0.030 \\
\hline Hypertension & $161(59 \cdot 6)$ & $62(54 \cdot 4)$ & 0.341 \\
\hline Diabetes mellitus & $121(44.8)$ & $21(18.4)$ & $<0.001$ \\
\hline Current or ex-smoking & $89(33.0)$ & $37(32.5)$ & 0.923 \\
\hline Chronic kidney disease & $26(9.6)$ & $2(1.8)$ & 0.007 \\
\hline Atrial fibrillation & $64(23.7)$ & $42(36.8)$ & 0.009 \\
\hline Ischemic heart disease & $119(44.1)$ & $20(17.5)$ & $<0.001$ \\
\hline Previous history of HF & $88(32.6)$ & $31(27.2)$ & 0.296 \\
\hline Implantable cardioverter defibrillator & $6(2.2)$ & $1(0.9)$ & 0.368 \\
\hline Cardiac resynchronization therapy & $1(0.4)$ & $1(0.9)$ & 0.528 \\
\hline Transfusion & $46(17.0)$ & $7(6.1)$ & 0.005 \\
\hline RRT during admission & $256(94.8)$ & $114(100)$ & 0.013 \\
\hline \multicolumn{4}{|l|}{ Medications at discharge } \\
\hline ACEi or ARB & $186(69.1)$ & $83(72.8)$ & 0.474 \\
\hline Aldosterone antagonist & $183(68.0)$ & $88(77.2)$ & 0.071 \\
\hline$\beta$-Blocker & $196(72.9)$ & $90(78.9)$ & 0.211 \\
\hline Nitrates & $74(27.4)$ & $19(16.7)$ & 0.025 \\
\hline Loop diuretics & $202(74.8)$ & $94(82.5)$ & 0.104 \\
\hline Digoxin & $37(13.7)$ & $25(21.9)$ & 0.045 \\
\hline Aspirin & $173(64.1)$ & $61(53 \cdot 5)$ & 0.053 \\
\hline Warfarin & $68(25.2)$ & $46(40.4)$ & 0.003 \\
\hline
\end{tabular}

Values are presented as mean \pm SD or number (\%).

HF, heart failure; NYHA, New York Heart Association; RRT, renal replacement therapy; ACEi, angiotensin-converting enzyme inhibitor; ARB, angiotensin receptor blocker.

${ }^{a} \mathrm{HF}$ with reduced ejection fraction was diagnosed as left ventricular ejection fraction $\leq 40 \%$. 
Table 2. Laboratory and echocardiographic findings

\begin{tabular}{|c|c|c|c|}
\hline Variable & Anemia $(\mathrm{n}=270)$ & No anemia $(n=114)$ & $p$ value \\
\hline \multicolumn{4}{|l|}{ Laboratory findings } \\
\hline Serum sodium, mEq/L & $136.9 \pm 4.0$ & $138.0 \pm 3.7$ & 0.008 \\
\hline Hyponatremia $^{\mathrm{a}}$ & $54(20.0)$ & $16(14.0)$ & 0.167 \\
\hline Initial level of hemoglobin, g/dL & $11.3 \pm 1.8$ & $14.5 \pm 1.5$ & $<0.001$ \\
\hline Lowest level of hemoglobin, g/dL & $10.1 \pm 1.6$ & $13.8 \pm 1.3$ & $<0.001$ \\
\hline Pre-discharge level of hemoglobin, g/dL & $11.1 \pm 1.4$ & $14.4 \pm 1.6$ & $<0.001$ \\
\hline Hemoglobin increase, g/dL & $1.0(0.3-1.9)$ & $0.5(0.1-1.1)$ & $<0.001$ \\
\hline Serum creatinine, mg/dL & $1.1(0.8-1.6)$ & $0.9(0.7-1.2)$ & $<0.001$ \\
\hline Alkaline phosphatase, U/L & $80(65-102)$ & $75(66-90)$ & 0.106 \\
\hline Lactate dehydrogenase, U/L & $535(438-652)$ & $505(426-585)$ & 0.875 \\
\hline NT-proBNP, pg/mL & $5,230(2,281-12,348)$ & $3,731(1,542-7,061)$ & 0.007 \\
\hline \multicolumn{4}{|l|}{ Echocardiographic findings } \\
\hline LVEDD, $\mathrm{mm}$ & $56.3 \pm 9.7$ & $57.4 \pm 10.9$ & 0.378 \\
\hline LVESD, mm & $44 \cdot 3 \pm 11.9$ & $45 \cdot 4 \pm 12.4$ & 0.438 \\
\hline LVEF & $39(29-51)$ & $37(23-49)$ & 0.146 \\
\hline LA diameter, mm & $46.9 \pm 8.9$ & $46.2 \pm 8.8$ & 0.499 \\
\hline $\mathrm{E}, \mathrm{m} / \mathrm{s}$ & $0.86(0.63-1.09)$ & $0.86(0.64-1.12)$ & 0.258 \\
\hline DT, ms & $158(117-208)$ & $155(119-199)$ & 0.204 \\
\hline $\mathrm{E} / \mathrm{E}^{\prime}$ & $17.9(13 \cdot 4-26.4)$ & $18.3(13.6-28.4)$ & 0.231 \\
\hline RVSP, mmHg & $43(34-55)$ & $42(33-50)$ & 0.234 \\
\hline
\end{tabular}

Values are presented as mean $\pm \mathrm{SD}$, number (\%), or median (interquartile range).

NT-proBNP, N-terminal pro-B-type natriuretic peptide; LVEDD, left ventricular end-diastolic dimension; LVESD, left ventricular end-systolic dimension; LVEF, left ventricular ejection fraction; LA, left atrium; E, peak early diastolic velocity; DT, deceleration time; E/E', ratio between peak early diastolic velocity and early diastolic tissue velocity; RVSP, right ventricular systolic pressure.

${ }^{\mathrm{a}}$ Hyponatremia was defined as a serum sodium level $<135 \mathrm{mEq} / \mathrm{L}$.
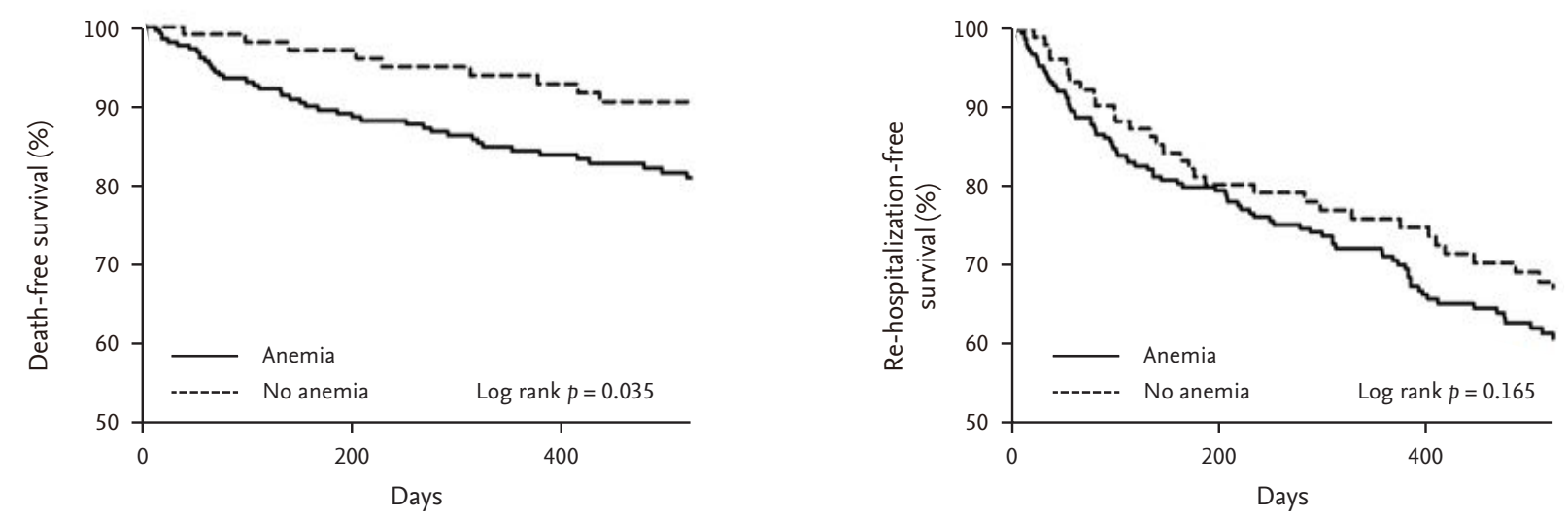

No. at risk

No. at risk

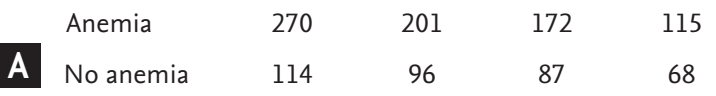

$\begin{array}{lcccc}\text { Anemia } & 270 & 173 & 139 & 86 \\ \text { B No anemia } & 114 & 81 & 70 & 53\end{array}$

Figure 1. Death-free survival (A) and heart failure re-hospitalization-free survival (B) on Kaplan-Meier analysis according to the presence of anemia during hospitalization. 
A

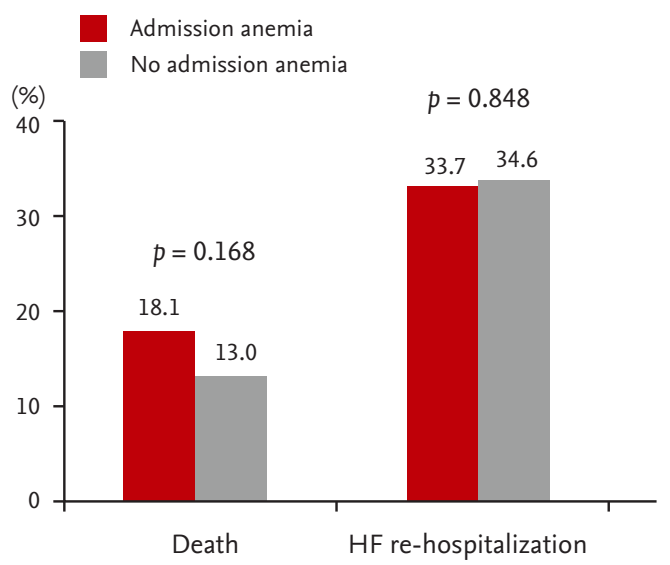

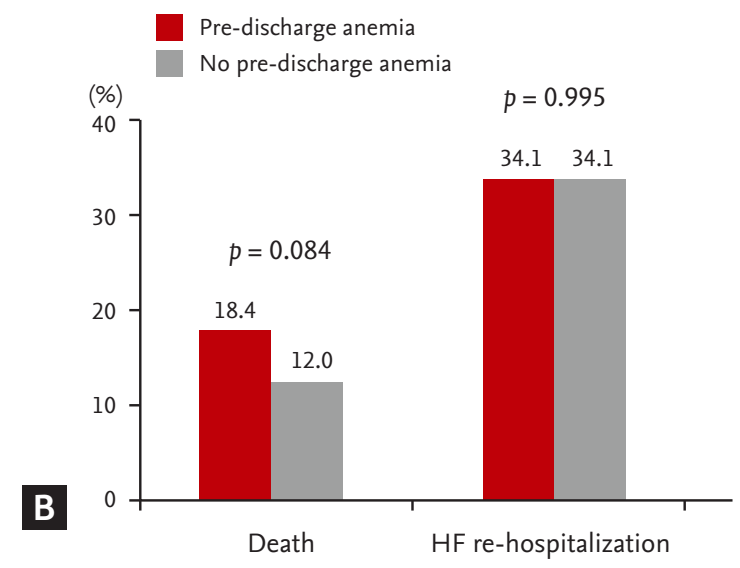

Figure 2. Death and heart failure (HF) re-hospitalization according to the presence of admission anemia (A) and pre-discharge anemia (B).

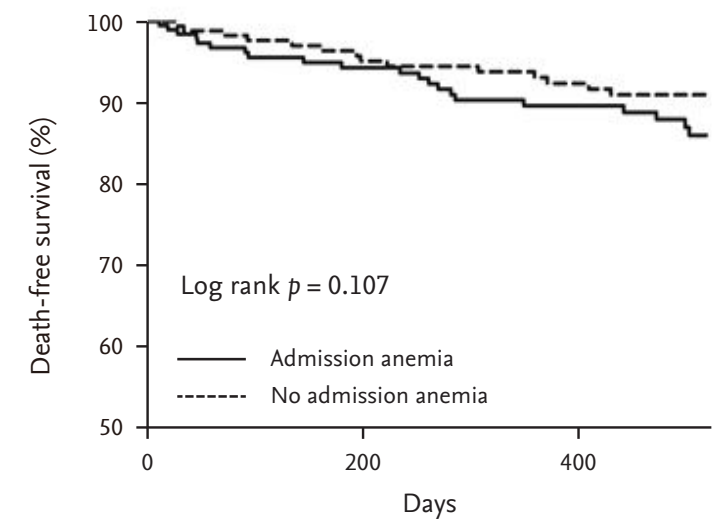

No. at risk

\begin{tabular}{|c|c|c|c|c|}
\hline Anemia & 199 & 147 & 126 & 83 \\
\hline No anemia & 185 & 150 & 133 & 109 \\
\hline
\end{tabular}

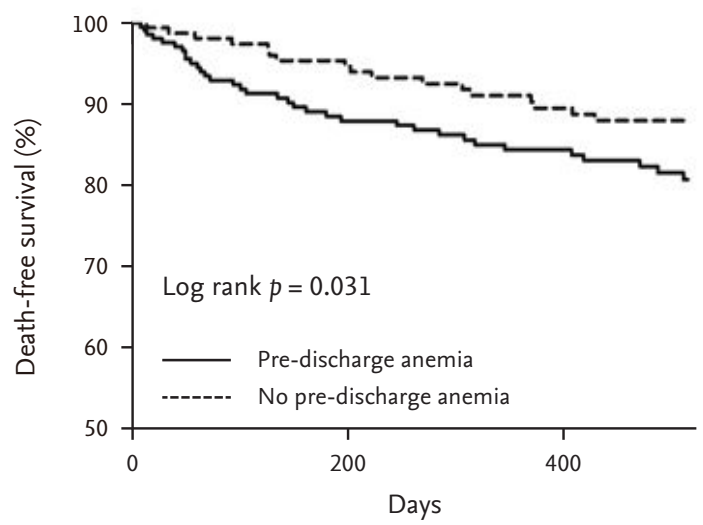

No. at risk

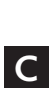
Anemia 217 159 138

93

No anemia

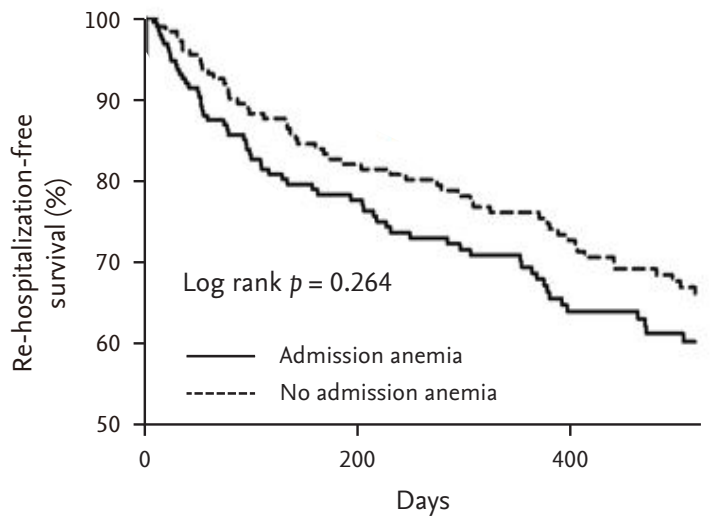

No. at risk

$\begin{array}{llccc}\text { Anemia } & 199 & 122 & 97 & 56 \\ \text { B No anemia } & 185 & 132 & 113 & 83\end{array}$

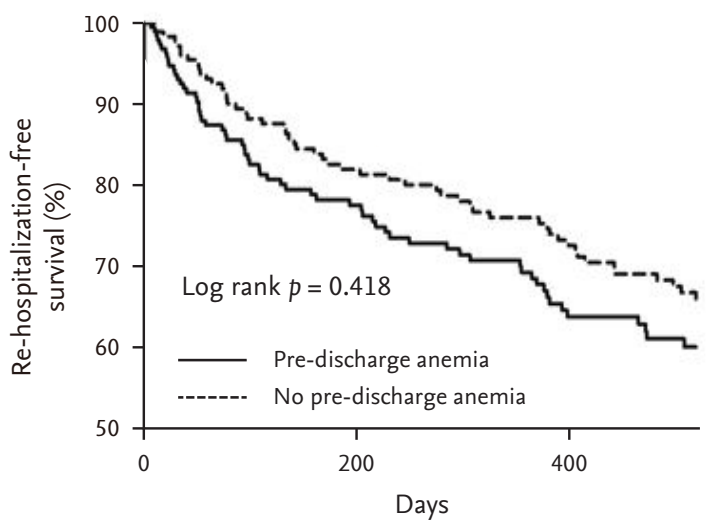

No. at risk

$\begin{array}{lcccc}\text { Anemia } & 217 & 137 & 111 & 68 \\ \text { No anemia } & 167 & 117 & 99 & 71\end{array}$

Figure 3. Death and heart failure re-hospitalization-free survival on Kaplan-Meier analysis according to the presence of admission anemia (A, B) and pre-discharge anemia (C, D). 


\section{RESULTS}

\section{Baseline characteristics}

Anemia was identified in 270 patients during hospitalization; anemia at admission in 199 patients (70.3\%); and pre-discharge anemia in 71 patients (29.7\%).

Baseline clinical characteristics are summarized in Table 1. The age and body mass index of the anemia group were higher and lower, respectively, compared to those of the no anemia group. The prevalence of HF with reduced ejection fraction was similar between groups. Diabetes mellitus, chronic kidney disease, and ischemic heart disease were significantly prevalent in the anemia group, whereas atrial fibrillation was significantly prevalent in the no anemia group. The degree of dyspnea, as measured by NYHA class, was significantly greater in the anemia group than in the no anemia group. Nitrates were more commonly prescribed in the anemia group, whereas digoxin and warfarin were more commonly prescribed in the no anemia group.

\section{Laboratory and echocardiographic findings}

Laboratory and echocardiographic findings are summarized in Table 2. The levels of Hgb throughout the hospitalization period were significantly lower, and the levels of serum creatinine and N-terminal pro-B-type natriuretic peptide were significantly higher, in the anemia group than in the no anemia group. Echocardiographic findings were not different between the groups.

\section{Clinical outcomes}

During clinical follow-up (528 days; interquartile range,
205 to 743 ), 60 patients (15.6\%) died and 131 patients (34.1\%) were re-hospitalized due to worsening of HF.

All-cause mortality and HF-related re-hospitalization were not different between the anemia and no anemia groups (17.8 vs. 10.5\%, $p=0.074 ; 35.2$ vs. $31.6 \%, p=0.496$, respectively). In Kaplan-Meier survival curve analysis, all-cause mortality was significantly higher in the anemia group than in the no anemia group (log-rank $p=$ 0.035) (Fig. 1), but HF-related re-hospitalization was not different between the groups.

Neither anemia at admission nor pre-discharge anemia was associated with all-cause mortality or re-hospitalization during clinical follow-up (Fig. 2). In Kaplan-Meier survival curve analysis, pre-discharge anemia showed a significant association with increased mortality compared to no pre-discharge anemia, but admission anemia was not associated with increased mortality during clinical follow-up (Fig. 3).

\section{Independent predictors of clinical outcomes}

Anemia during hospitalization was a significant predictor of mortality (adjusted hazard ratio [HR], 1.74; 95\% confidence interval [CI], 1.03 to $3.01 ; p=0.039$ ). Pre-discharge anemia was also an independent predictor of future mortality (adjusted HR, 1.68; 95\% CI, 1.01 to 2.82; p $=0.048$ ), but anemia at admission was not a predictor of increased mortality (adjusted HR, 1.12; 95\% CI, 0.61 to 2.07; $p=0.719$ ) (Table 3). Neither admission nor pre-discharge anemia predicted HF-related re-hospitalization. Old age ( $\geq 65$ years), diabetes mellitus, hypotension, and atrial fibrillation were also independent predictors of mortality, but old age ( $\geq 65$ years) was the only

Table 3. The association between the timing of anemia and clinical outcomes

\begin{tabular}{|c|c|c|c|c|}
\hline \multirow{2}{*}{ Variable } & \multicolumn{2}{|c|}{ Unadjusted } & \multicolumn{2}{|c|}{ Adjusted } \\
\hline & $\operatorname{HR}(95 \% \mathrm{CI})$ & $p$ value & $\operatorname{HR}(95 \% \mathrm{CI})$ & $p$ value \\
\hline \multicolumn{5}{|l|}{ All-cause mortality } \\
\hline Anemia during hospitalization (vs. no anemia) & $1.95(1.04-3.67)$ & 0.039 & $1.74(1.03-3.01)$ & 0.039 \\
\hline Admission anemia (vs. no admission anemia) & $1.67(0.99-2.79)$ & 0.054 & $1.12(0.61-2.07)$ & 0.719 \\
\hline Discharge anemia (vs. no discharge anemia) & $1.79(1.05-3.07)$ & 0.033 & $1.68(1.01-2.82)$ & 0.048 \\
\hline \multicolumn{5}{|l|}{ Readmission due to ADHF } \\
\hline Anemia during hospitalization (vs. no anemia) & $1.31(0.89-1.93)$ & 0.167 & $0.92(0.59-1.42)$ & 0.707 \\
\hline Admission anemia (vs. no admission anemia) & $1.22(0.86-1.72)$ & 0.265 & $0.90(0.59-1.37)$ & 0.620 \\
\hline Discharge anemia (vs. no discharge anemia) & $1.15(0.82-1.63)$ & 0.418 & $0.81(0.53-1.24)$ & 0.325 \\
\hline
\end{tabular}

HR, hazard ratio; CI, confidence interval; ADHF, acute decompensated heart failure. 
Table 4. Predictors for adverse clinical outcomes

\begin{tabular}{|c|c|c|}
\hline Variable & Adjusted HR (95\% CI) & $p$ value \\
\hline \multicolumn{3}{|l|}{ All-cause mortality } \\
\hline Discharge anemia (vs. no discharge anemia) & $1.68(1.01-2.82)$ & 0.048 \\
\hline Age 65 years or more & $6.22(1.86-20.80)$ & 0.003 \\
\hline NYHA classification 3 or 4 & $0.81(0.41-1.62)$ & 0.553 \\
\hline Systolic blood pressure $\leq 100 \mathrm{mmHg}$ & $2.33(1.24-4.79)$ & 0.021 \\
\hline Diabetes mellitus & $2.01(1.07-3.79)$ & 0.031 \\
\hline Ischemic etiology of ADHF & $1.42(0.76-2.65)$ & 0.276 \\
\hline Atrial fibrillation & $2.59(1.37-4.90)$ & 0.003 \\
\hline Serum creatinine & $1.01(0.77-1.34)$ & 0.930 \\
\hline Serum sodium < $135 \mathrm{mEq} / \mathrm{L}$ & $1.13(0.56-2.27)$ & 0.741 \\
\hline LVEF & $1.01(0.99-1.03)$ & 0.408 \\
\hline \multicolumn{3}{|l|}{ Readmission due to ADHF } \\
\hline Discharge anemia (vs. no discharge anemia) & $0.81(0.53-1.24)$ & 0.325 \\
\hline Age 65 years or more & $1.82(1.11-2.99)$ & 0.018 \\
\hline NYHA classification 3 or 4 & $1.05(0.65-1.70)$ & 0.838 \\
\hline Systolic blood pressure $\leq 100 \mathrm{mmHg}$ & $0.90(0.53-1.55)$ & 0.710 \\
\hline Diabetes mellitus & $0.94(0.60-1.48)$ & 0.793 \\
\hline Ischemic etiology of ADHF & $1.13(0.73-1.74)$ & 0.592 \\
\hline Atrial fibrillation & $1.22(0.78-1.90)$ & 0.391 \\
\hline Serum creatinine & $1.08(0.88-1.32)$ & 0.464 \\
\hline Serum sodium < $135 \mathrm{mEq} / \mathrm{L}$ & $1.57(0.97-2.52)$ & 0.064 \\
\hline LVEF & $1.00(0.99-1.01)$ & 0.826 \\
\hline
\end{tabular}

HR, hazard ratio; CI, confidence interval; NYHA, New York Heart Association; ADHF, acute decompensated heart failure; LVEF, left ventricular ejection fraction.

independent predictor of HF-related re-hospitalization (Table 4).

\section{DISCUSSION}

The authors investigated the impact of the timing of anemia during hospitalization on future clinical outcomes in surviving ADHF patients from an index HF, and the principal findings of the present study were as follows: (1) anemia is highly prevalent (70.3\%) and a predictor of future mortality in patients with ADHF; (2) pre-discharge anemia, rather than anemia at admission, is an independent predictor of mortality in patients with ADHF after surviving discharge; and (3) neither anemia at admission nor pre-discharge anemia is associated with HF-related re-hospitalization in patients with ADHF.

Numerous studies have evaluated the association between anemia and clinical outcomes in patients with HF; however, most studies examined the prognostic impact of anemia in chronic HF [15-18]. Furthermore, studies on anemia in ADHF patients have examined the prognostic impact of anemia at a single time point, generally at the time of presentation [1-4]. To the best of our knowledge, few studies have evaluated the clinical impact of anemia at different times during hospitalization. Stojcevski et al. [6] showed that discharge Hgb was related to re-hospitalization but not mortality in patients with ADHF. Other study reported that a change in the level of Hgb during hospitalization was independently associated with clinical outcomes in ADHF [7]. We found that only anemia at discharge increased all-cause mortality; however, neither anemia at admission nor 
discharge affected the readmission rate due to worsening of ADHF. In contrast with the results of the abovementioned study [6], discharge anemia was not associated with readmission due to ADHF in our study, and this difference in clinical outcome between the two studies may be related to several factors, such as different LVEF or ethnicity of the sample. Because both are small retrospective studies, works with larger, real-world registries or randomized trials are needed to further explore this issue. In the current study, transfusion was performed in 53 patients $(13.8 \%)$, and more patients with anemia received transfusion than those without anemia $(17.0 \%$ vs. $6.1 \%, p=0.005)$. Transfusion also affected the study results similarly to the original analysis, in terms of death (HR, 1.89 for transfusion; 95\% CI, 1.02 to $3.50 ; p=0.042$ ) and HF-related readmission (HR, 1.05; 95\% CI, 0.63 to 1.74; $p=0.862$ ). However, we believe that transfusion itself was not a predictive factor for clinical outcomes, but rather a supportive treatment for anemia. Therefore, we did not include it in the multivariate model.

Reasons for higher mortality in ADHF patients with discharge anemia were probably multifactorial (e.g., a higher prevalence of ischemic heart disease in the anemia group). Patients with coronary artery disease should be prescribed an antiplatelet indefinitely, irrespective of the recanalization state of the infarct-related artery, thereby triggering iron deficiency anemia [19,20]. However, admission anemia did not influence mortality in our study, emphasizing the importance of discharge anemia in ADHF. The main reason for this is probably that patients could not manage anemia after discharge. Current guidelines for ADHF do not set an optimal target range of $\mathrm{Hgb}$ in patients with $\mathrm{ADHF}$ and anemia during hospitalization [10,11]. Furthermore, hemoconcentration also positively affects clinical outcomes in $\operatorname{ADHF}[7,21,22]$. In one study, hemoconcentration occurring late during hospitalization was only associated with improved survival in patients with ADHF compared to early hemoconcentration, and this finding intensified the results of the current study [23]. However, physicians may not be able to transfuse a sufficient amount of red blood cell to raise the $\mathrm{Hgb}$ level up to an anemic range in real world clinical practice, because of insurance issues or volume overload. Therefore, the optimal target range of Hgb should be established using large-scale registries in these high-risk patients.
The main limitation of the present study was that it used a retrospective, single-center design and included a relatively small number of patients. Second, management for anemia was not standardized in our study population. However, patients were managed with transfusion to raise the level of $\mathrm{Hgb}$ above $7 \mathrm{~g} / \mathrm{dL}$ according to the treatment guidelines for anemia, and not for HF [24]. Third, we could not evaluate the revascularization state of ischemic heart disease in our study population. Considering the high prevalence $(36.2 \%)$ of ischemic heart disease as an etiology of ADHF, revascularization of an infarct-related artery may have influenced our clinical outcomes. Fourth, there were no laboratory data on iron deficiency. Therefore, anemia was probably partly associated with hemodilution. The type of anemia, such as iron deficiency anemia, anemia with chronic disease, or anemia with acute blood loss, was not fully evaluated. Finally, although we adjusted for multiple confounding factors, we cannot exclude the possibility of residual confounding factors, as a result of the presence of an unmeasured confounder or measurement errors in the included factors.

\section{KEY MESSAGE}

1. The presence of anemia during hospitalization was associated with increased mortality, but not the re-hospitalization rate, in patients with acute decompensated heart failure (ADHF).

2. Anemia at admission did not predict increased mortality or re-hospitalization rate. Only anemia at discharge time was associated with increased mortality in patients with ADHF.

3. The identification and optimal management of anemia during hospitalization will be important in patients with ADHF to reduce mortality.

\section{Conflict of interest}

No potential conflict of interest relevant to this article was reported.

\section{Acknowledgments}

This study was supported by a grant of the Korea Health Technology R\&D Project through the Korea Health Industry Development Institute (HI15Co498), 
and grants of National Research Foundation of Korea, funded by the Korea government (2015 $\mathrm{M}_{3} \mathrm{~A}_{9} \mathrm{~B}_{40} \mathrm{O}_{106}$, 2015M3A9B4066496, 2016R1DiA1A09917796), by the Bio \& Medical Technology Development Program of the NRF funded by the Korean government, MSIP (2017M3A9E8023001) and by a grant (CRI13901-22.1) Chonnam National University Hospital Biomedical Research Institute.

\section{REFERENCES}

1. Young JB, Abraham WT, Albert NM, et al. Relation of low hemoglobin and anemia to morbidity and mortality in patients hospitalized with heart failure (insight from the OPTIMIZE-HF registry). Am J Cardiol 2008;101:223-230.

2. Hamaguchi S, Tsuchihashi-Makaya M, Kinugawa S, et al. Anemia is an independent predictor of long-term adverse outcomes in patients hospitalized with heart failure in Japan. A report from the Japanese Cardiac Registry of Heart Failure in Cardiology (JCARE-CARD). Circ J 2009;73:19011908.

3. Caughey MC, Avery CL, Ni H, et al. Outcomes of patients with anemia and acute decompensated heart failure with preserved versus reduced ejection fraction (from the ARIC study community surveillance). Am J Cardiol 2014;114:1850-1854.

4. Ezekowitz JA, McAlister FA, Armstrong PW. Anemia is common in heart failure and is associated with poor outcomes: insights from a cohort of 12065 patients with new-onset heart failure. Circulation 2003;107:223-225.

5. Kaiafa G, Kanellos I, Savopoulos C, Kakaletsis N, Giannakoulas G, Hatzitolios AI. Is anemia a new cardiovascular risk factor? Int J Cardiol 2015;186:117-124.

6. Stojcevski B, Celic V, Navarin S, et al. The use of discharge haemoglobin and NT-proBNP to improve short and longterm outcome prediction in patients with acute heart failure. Eur Heart J Acute Cardiovasc Care 2017;6:676-684.

7. Damluji AA, Macon C, Fox A, et al. The association between in-hospital hemoglobin changes, cardiovascular events, and mortality in acute decompensated heart failure: results from the ESCAPE trial. Int J Cardiol 2016;222:531-537.

8. van der Meer P, Postmus D, Ponikowski P, et al. The predictive value of short-term changes in hemoglobin concentration in patients presenting with acute decompen- sated heart failure. J Am Coll Cardiol 2013;61:1973-1981.

9. Choi DJ, Han S, Jeon ES, et al. Characteristics, outcomes and predictors of long-term mortality for patients hospitalized for acute heart failure: a report from the Korean Heart Failure Registry. Korean Circ J 2011;41:363-371.

10. Ponikowski P, Voors AA, Anker SD, et al. 2016 ESC guidelines for the diagnosis and treatment of acute and chronic heart failure: the task force for the diagnosis and treatment of acute and chronic heart failure of the European Society of Cardiology (ESC) developed with the special contribution of the Heart Failure Association (HFA) of the ESC. Eur Heart J 2016;37:2129-2200.

11. Yancy CW, Jessup M, Bozkurt B, et al. 2013 ACCF/AHA guideline for the management of heart failure: a report of the American College of Cardiology Foundation/American Heart Association Task Force on Practice Guidelines. J Am Coll Cardiol 2013;62:e147-e239.

12. Nutritional anaemias. Report of a WHO scientific group. World Health Organ Tech Rep Ser 1968;405:5-37.

13. Yoon HJ, Kim KH, Kim JY, et al. Chemotherapy-induced left ventricular dysfunction in patients with breast cancer. J Breast Cancer 2016;19:402-409.

14. Lang RM, Badano LP, Mor-Avi V, et al. Recommendations for cardiac chamber quantification by echocardiography in adults: an update from the American Society of Echocardiography and the European Association of Cardiovascular Imaging. J Am Soc Echocardiogr 2015;28:1-39.

15. Ebner N, Jankowska EA, Ponikowski P, et al. The impact of iron deficiency and anaemia on exercise capacity and outcomes in patients with chronic heart failure. Results from the Studies Investigating Co-morbidities Aggravating Heart Failure. Int J Cardiol 2016;205:6-12.

16. von Haehling S, van Veldhuisen DJ, Roughton M, et al. Anaemia among patients with heart failure and preserved or reduced ejection fraction: results from the SENIORS study. Eur J Heart Fail 2011;13:656-663.

17. Groenveld HF, Januzzi JL, Damman K, et al. Anemia and mortality in heart failure patients a systematic review and meta-analysis. J Am Coll Cardiol 2008;52:818-827.

18. Yamauchi T, Sakata Y, Takada T, et al. Prognostic impact of anemia in patients with chronic heart failure: with special reference to clinical background: report from the CHART-2 study. Circ J 2015;79:1984-1993.

19. Amsterdam EA, Wenger NK, Brindis RG, et al. 2014 AHA/ ACC guideline for the management of patients with non-ST-elevation acute coronary syndromes: a report 
of the American College of Cardiology/American Heart Association Task Force on Practice Guidelines. J Am Coll Cardiol 2014;64:e139-e228.

20. American College of Emergency Physicians; Society for Cardiovascular Angiography and Interventions, O'Gara PT, et al. 2013 ACCF/AHA guideline for the management of ST-elevation myocardial infarction: a report of the American College of Cardiology Foundation/American Heart Association Task Force on Practice Guidelines. J Am Coll Cardiol 2013;61:e78-e140.

21. Oh J, Kang SM, Kim IC, et al. The beneficial prognostic value of hemoconcentration is negatively affected by hyponatremia in acute decompensated heart failure: data from the Korean Heart Failure (KorHF) Registry. J Cardi- ol 2017;69:790-796.

22. Oh J, Kang SM, Hong N, et al. Hemoconcentration is a good prognostic predictor for clinical outcomes in acute heart failure: data from the Korean Heart Failure (KorHF) Registry. Int J Cardiol 2013;168:4739-4743.

23. Testani JM, Brisco MA, Chen J, McCauley BD, Parikh CR, Tang WH. Timing of hemoconcentration during treatment of acute decompensated heart failure and subsequent survival: importance of sustained decongestion. J Am Coll Cardiol 2013;62:516-524.

24. Carson JL, Guyatt G, Heddle NM, et al. Clinical practice guidelines from the AABB: red blood cell transfusion thresholds and storage. JAMA 2016;316:2025-2035. 